\title{
Some non-standard Sobolev spaces, interpolation and its application to PDE
}

\author{
González, María del Mar* and Gualdani, Maria Pia**
}

\begin{abstract}
We consider some nonstandard Sobolev spaces in one dimension, in which functions have different regularity in different subsets. Those spaces are useful in the study of some nonlinear elliptic and parabolic equations where the nonlinearity is highly degenerate and, in order to handle this degeneracy, additional partial regularity in subsets is required. The authors give denseness and interpolation results that are needed for the PDE theory to apply.
\end{abstract}

\section{Introduction}

Let $\Omega=[-1,1] \subset \mathbb{R}$ be an interval and $I$ a given subset of $\Omega$. We consider the following nonlinear parabolic equation for $f(x, t)$ :

$$
\left\{\begin{array}{l}
f_{t}-\Delta f=\mu\left(f_{\mid I}, f_{\left.x\right|_{I}}, f_{\left.x x\right|_{I}}\right), \quad x \in \Omega, t>0 \\
f(\cdot, 0)=f_{I}, \\
f(-1, t)=f(1, t)=0, \quad \text { for all } t>0,
\end{array}\right.
$$

where $\mu$ is a distribution with support compactly contained in $\Omega \backslash I$. The non-linearity may be a very general one and could even include a free boundary for the problem.

In the case where the non-linearity $\mu$ satisfies some boundedness conditions, it is well known that the operator $\Delta+\mu$ shares the same spectral properties as the Laplacian operator $\Delta$ (see for example [5] and [3]). Thus semigroup theory can be applied to produce a solution of (1.1), given suitable functional spaces. More precisely, we need to find three function spaces

$$
Z \subset Y \subset X,
$$

where $Y:=X^{\alpha}$ is taken as the interpolation space between $X$ and $Z$, for any $0<\alpha<1$, and such that

$$
\begin{aligned}
& \Delta: X \rightarrow X, \quad \text { unbounded linear operator, closed, } \\
& -\Delta \text { is a sectorial operator in } X \\
& Z=D(\Delta), \quad \text { densely defined in } X \\
& \mu: Y \rightarrow X, \quad \text { bounded operator. }
\end{aligned}
$$

Once we achieve this functional space setting, local existence and uniqueness for (1.1) follows from standard arguments in semigroup theory (see Theorem 3.3.3. and 5.1.1. in [5]):

\footnotetext{
${ }^{1} 2000$ Mathematics Subject Classification: 35K05, 35S05, 26A33, 46B70

*Universitat Politècnica de Catalunya, ETSEIB - Departament de Matemàtica Aplicada I, Av. Diagonal 647, 08028 Barcelona, SPAIN. Email: mar.gonzalez@upc.edu

** Department of Mathematics, University of Texas at Austin, 1 University Station C1200 Austin, Texas 78712, USA. Email: gualdani@math.utexas.edu
} 
Theorem 1.1. Assume that $\mu: U \subset Y \rightarrow X$ is locally Lipschitz in $U$, i.e, given any $f \in U$, there exists a smaller neighborhood $f \in V \subset U$ such that for all $f_{1}, f_{2} \in V$ we have that

$$
\left\|\mu\left(f_{1}\right)-\mu\left(f_{2}\right)\right\|_{X} \leq L\left\|f_{1}-f_{2}\right\|_{Y}
$$

for some constant $L>0$. Then, for any $f_{I} \in U \subset Y$, there exists $T=T\left(f_{I}\right)>0$ such that (1.1) has a unique solution $f(x, t)$ for all times $t \in[0, T)$ with initial value $f(\cdot, 0)=f_{I}$.

If, in addition, the origin is an equilibrium point, $\|\mu(f)\|_{X}=o\left(\|f\|_{Y}\right)$ when $\|f\|_{Y} \rightarrow 0$, and the spectrum of $(-\Delta)$ is contained in the region $\{\operatorname{Re}(z)>\beta\}$ for some $\beta>0$, then the origin is uniformly asymptotically stable.

However, in order to satisfy all the conditions in (1.2)-(1.5), the choice of functional spaces is delicate and based on the shape of $\mu$; this is the main purpose of the present paper. Suppose that, as in the case of (1.1), the nonlinear part of the operator is a very rough distribution in $H^{r}(\Omega)$ for some $r \in \mathbb{R}$ (in our applications, $r<0$ ), with coefficients depending on the value of the function $f$ and its derivatives on a smaller subset $I$ of $\Omega$. The distribution has support in the complementary set of $I$.

Thus, in order to satisfy the properties (1.2)-(1.5), one can simply choose $X=H^{r}, r \in \mathbb{R}$, so that $\Delta: H^{r} \rightarrow H^{r}, D(\Delta)=H^{r+2}, Y=H^{r+2 \alpha}$ for any $0<\alpha<1$. However, in order to have the Lipschitz property for $\mu$, more regularity for the function $f$ is required in the subset $I$. Therefore, we will ask $X$ to be

$$
X=H^{r} \cap H_{\phi}^{s}
$$

for $r<s, s>0$, that is defined through the norm

$$
\|f\|_{X}:=\|f\|_{H^{r}}+\|\phi f\|_{H^{s}} .
$$

Here $\phi$ is a smooth cutoff function supported on $I$. Given this $X$, the domain of the Laplacian operator becomes, naively,

$$
Z=H^{r+2} \cap H_{\phi}^{s+2},
$$

with a slight modification as explained below in Proposition 2.1.

The main issue remains now to find the intermediate space $Y$. This space is important because, given $f_{I} \in Y$, any solution $f$ of (1.1) will belong to $Y$ for any $t>0$, as stated in Theorem 1.1. It is given as the (real) interpolation between $Z$ and $X$.

Summarizing, the aim of the present paper to provide the functional space setting required for the application of semigroup tools for the problem (1.1), in order to obtain local existence and stability of equilibrium points. While semigroup methods are somewhat standard, the main novelty here is the use of our non-standard intersection spaces, that allow to handle certain types of non-linearities. We have not found any reference in the literature that deals with these intersection spaces. In particular, we concentrate on the issue of denseness of $Z$ in $X$ (section 2) and on interpolation between $X$ and $Z$ (section 3 ).

An application of this result can be found in [4]. There the authors study the following free boundary problem, coming from a model in formation of prices: find $f(x, t)$ and $p(t)$ solution of

$$
\left\{\begin{array}{l}
\partial_{t} f-\partial_{x x} f=-\partial_{x} f(p(t), t)\left[\delta_{x=p(t)-a}-\delta_{x=p(t)+a}\right], \quad x \in[-1,1], t>0 \\
\dot{p}(t)=-\frac{f_{x x}(p(t), t)}{f_{x}(p(t), t)} \\
f(x, 0)=f_{I}(x) \text { in }[-1,1] \\
p(0)=p_{I}
\end{array}\right.
$$

Global existence for this problem was shown in [2] using a different point of view. However, stability was not achieved with the methods there. 
Another example is precisely the linearized operator of the previous problem around an equilibrium point:

$$
\partial_{t} f=f_{x x}-f_{x}\left(p_{1}\right)\left[\delta_{x=p_{1}-a}-\delta_{x=p_{1}+a}\right]+f\left(p_{1}\right)\left[\delta_{x=p_{1}-a}^{\prime}-\delta_{x=p_{1}+a}^{\prime}\right],
$$

that is also considered in [4].

\section{$2 \quad$ Function spaces}

Periodic functions on $\mathbb{R}$ with period on the interval $[-1,1]$ can be considered as defined on the onedimensional torus $\mathbb{T}^{1}$. Such functions have Fourier expansion as

$$
f \sim \sum \hat{f}_{n} e_{n}
$$

where $\left\{e_{n}\right\}$ is an orthonormal basis in $\mathbb{T}^{1}$ and $\hat{f}_{n}$ are the Fourier coefficients of $f$. The Hilbert space $H^{p}\left(\mathbb{T}^{1}\right)$ for any $p \in \mathbb{R}$ is given by the norm

$$
\|f\|_{H^{p}}:=\sum\left(1+n^{2}\right)^{p}\left|\hat{f}_{n}\right|^{2}
$$

Let $\phi$ be a smooth cutoff function with support on $[-1 / 2,1 / 2]$, indentically one on $[-1 / 4,1 / 4]$. We also consider the space $H_{\phi}^{p}\left(\mathbb{T}^{1}\right)$ given by the norm

$$
\|f\|_{H_{\phi}^{p}}:=\|\phi f\|_{H^{p}} .
$$

Given $r \in \mathbb{R}, s>0, r<s$, we will work with the intersection space $X:=H^{r} \cap H_{\phi}^{s}$, with norm defined as in (1.6). From now on, we will drop the $\mathbb{T}^{1}$ in the notation of the spaces and norms.

Consider now the Laplacian operator acting on $X$,

$$
\Delta f=\sum \lambda_{n} \hat{f}_{n} e_{n}, \quad \lambda_{n} \sim-n^{2} .
$$

here $\lambda_{n}$ are the eigenvalues of the Laplacian, that are negative except the value zero, that can be deleted if we exclude the constant functions.

Define also the space

$$
Z=H^{r+2} \cap H_{\hat{\phi}}^{s+2}
$$

where $\hat{\phi}$ is another cutoff function such that $\hat{\phi} \equiv 1$ on the support of $\phi$. The following proposition justifies the choice of $Z$ as the domain of $L$.

Proposition 2.1. With this choice of function spaces,

$$
X=H^{r} \cap H_{\phi}^{s}, \quad Z=H^{r+2} \cap H_{\hat{\phi}}^{s+2},
$$

it holds:

1. $L: Z \rightarrow X$ is a bounded operator.

2. $Z$ is dense in $X$.

Proof. For the first assertion, just note that the choice of the cutoff functions $\phi$ and $\hat{\phi}$ implies:

$$
\phi \Delta(\hat{\phi} f)=\phi \Delta(f) .
$$

For the second, we will prove that periodic $\mathcal{C}^{\infty}$-functions are dense in $X$ : given $f_{0} \in X$ and $\epsilon>0$, we will find a smooth sequence $f_{\epsilon}$ such that $\left\|f_{\epsilon}-f_{0}\right\|_{X}<\epsilon$. 
The idea is the following: given a collection $\mathcal{U}$ of overlapping intervals covering $[-1,1]$, we construct a good approximation for $f_{0}$ in each subintervals of $\mathcal{U}$. Consider for example

$$
\mathcal{U}=\{[-1,-1 / 2],[-5 / 8,-1 / 4],[-3 / 8,3 / 8],[1 / 4,5 / 8],[-1 / 2,1]\} .
$$

The convergence in the whole interval $[-1,1]$ follows from the convergence in the single subintervals by a partition of unity argument.

The convergence in $H^{r}$ is guaranteed by the regularity of the heat equation. Indeed, let $f(x, t)$ be the solution of the heat equation $f_{t}=\Delta f$ with initial condition $f_{0}$. Then

$$
f(x, t)=\sum e^{\lambda_{n} t} \hat{f}_{n} e_{n}
$$

satisfies $f \rightarrow f_{0}$ in $H^{r}$ when $t \rightarrow 0$. Moreover, also $f \rightarrow f_{0}$ in $H^{s}$ on $[-3 / 8,3 / 8]$, away from the vanishing points of the cutoff $\phi$.

The delicate part is the convergence near $x= \pm 1 / 2$. For that, on the subinterval $[-5 / 8,-1 / 4]$ we regularize the function $f_{0}$ as $f_{\epsilon}=\theta_{\epsilon} * f_{0}$ where $\theta$ is a mollifier with support in $(0,1)$ and $\theta_{\epsilon}(x)=\frac{1}{\epsilon} \theta(x / \epsilon)$, with supp $\theta_{\epsilon}=(0, \epsilon)$. Since $f_{0} \in H^{r}$, it is known that $f_{\epsilon} \rightarrow f_{0}$ in $H^{r}$, while it is not clear that $\phi f_{\epsilon}$ convergens to $\phi f_{0}$ because of the inability of switching the order of convolution and cutoff.

This problem can be handled as follows: by assumption, $\phi f_{0} \in H^{s}$. Consider a small shifting, $\phi(x-$ t) $f_{0}(x) \in H^{s}$ for $t \in(0, \epsilon)$, so that $\phi(x) f_{0}(x+t) \in H^{s}$. Thus when we average with respect to the weight $\theta_{\epsilon}$, then the function $\phi(x) f_{\epsilon}(x)$, defined as

$$
\phi(x) f_{\epsilon}(x)=\phi(x) \int_{0}^{\epsilon} f_{0}(x+t) \theta_{\epsilon}(t) d t,
$$

still belongs to $H^{s}$ with norm uniformly bounded with respect to $\epsilon$, depending on $\left\|\phi f_{0}\right\|_{H^{s}}$.

We claim that $\phi f_{\epsilon}$ is very close to $\phi f_{0}$ on $[-5 / 8,-1 / 4]$. First note that for all $\delta>0$, there exists a function $\psi \in \mathcal{C}^{\infty}$ with compact support in the interval $[-5 / 8,-1 / 4]$ such that $\left\|\phi\left(f_{0}-\psi\right)\right\|_{H^{s}} \leq \delta / 3$. Consider now $\psi_{\epsilon}:=\theta_{\epsilon} * \psi$. It holds

$$
\left\|\phi f_{\epsilon}-\phi f_{0}\right\|_{H^{s}} \leq\left\|\phi\left(f_{\epsilon}-\psi_{\epsilon}\right)\right\|_{H^{s}}+\left\|\phi\left(\psi_{\epsilon}-\psi\right)\right\|_{H^{s}}+\left\|\phi\left(f_{0}-\psi\right)\right\|_{H^{s}} .
$$

The first term in the sum can be estimated as in (2.1), and in particular, it can be uniformly bounded by $\left\|\phi\left(f_{0}-\psi\right)\right\|_{H^{s}}<\delta / 3$. The second term can be made small thanks to a standard property of mollifiers, and the third is bounded by hypothesis of the initial data.

Thus we have that $\left\|\phi f_{\epsilon}-\phi f_{0}\right\|_{H^{s}} \leq \delta$ for $\epsilon$ small enough, as desired.

\section{Interpolation}

Consider the unbounded linear operator $\Delta: X \rightarrow X$, with dense domain $Z=D(X)$. We seek to give a characterization of the intermediate space $Y:=D\left((-\Delta)^{\alpha}\right)=X^{\alpha}$, for $0<\alpha<1$, that is the domain of a fractional power of the Laplacian operator.

In the case that the function space is $X:=H^{p}$ for $p \in \mathbb{R}$, everything can be written explicitly using Fourier series. The operator $(-\Delta)^{\alpha}$ for $0<\alpha<1$ is the fractional Laplacian defined by semigroup theory as

$$
(-\Delta)^{\alpha}=\int_{0}^{\infty} t^{\alpha-1}(-\Delta)(t I+(-\Delta))^{-1} d t,
$$

and it can be written using Fourier series as

$$
(-\Delta)^{\alpha} f=\sum_{n} \hat{f}_{n} \lambda_{n}^{\alpha} e_{n}(x)
$$


where the domain of $(-\Delta)^{\alpha}$ is

$$
X^{\alpha}=H^{2 \alpha+p} .
$$

For a general space $X$, the following characterization holds:

Theorem 3.1 (Theorem 1.18.10. in [14]). Let $L$ be positive self-adjoint operator. If $\gamma_{1}$ and $\gamma_{2}$ are two complex numbers, $0 \leq \Re \gamma_{1}<\Re \gamma_{2}<\infty$ and $0<\alpha<1$, then

$$
\left(D\left(L^{\gamma_{1}}\right), D\left(L^{\gamma_{2}}\right)\right)_{\alpha, 2}=D\left(L^{\gamma_{1}(1-\alpha)+\gamma_{2} \alpha}\right) .
$$

In particular,

$$
D\left(L^{\alpha}\right)=(X, D(L))_{\alpha, 2}
$$

Thus we are interested on this interpolation space between $X=H^{r} \cap H_{\phi}^{s}$ and $Z:=H^{r+2} \cap H_{\phi}^{s+2}$.

Note that, for simplicity and without loss of generality for the purposes of this work, we are taking the same cutoff function for both $X$ and $Z$, which differs from the previously defined $Z$ defined in Proposition 2.1. For the original case, see the remark at the end.

For any $0<\alpha<1$, we consider the (real) interpolation space

$$
Y:=(X, Z)_{\alpha, 2}
$$

(see [12], [13], [14] for the precise definitions). Although $Y$ cannot be characterized exactly due to the non-standard form of the space $X$, we will prove that

Theorem 3.2. There exists two function spaces $\hat{Y}$ and $\tilde{Y}$ such that

$$
\hat{Y} \subset Y \subset \tilde{Y} .
$$

Here $\hat{Y}$ is defined in (3.4), and $\tilde{Y}$ in (3.6).

Finding $\hat{Y}$ involves understanding pseudo-differential operators, while for the space $\tilde{Y}$ a careful look at interpolation theory is required.

\subsection{Characterization of $\tilde{Y}$}

The definition of (real) interpolation of spaces uses interpolation functors (see [13], section 1.6.2). Let $A_{0}$ and $A_{1}$ be two Banach spaces. We say that $\left\{A_{0}, A_{1}\right\}$ is an interpolation couple if there exists a linear Hausdorff space $A$ such that both $A_{0}$ and $A_{1}$ are linearly and continuously embedded in $A$. Then the spaces $A_{0} \cap A_{1}$ and $A_{0}+A_{1}$ with

$$
A_{0}+A_{1}=\left\{a \in A: a=a_{0}+a_{1} \text { for some } a_{0} \in A_{0}, a_{1} \in A_{1}\right\}
$$

are well defined. For each $t>0$, we consider the Peetre's $K$-functional as

$$
K(t, a)=\inf _{\left(a_{0}, a_{1}\right)}\left\{\left\|a_{0}\right\|_{A_{0}}+t\left\|a_{1}\right\|_{A_{1}}\right\},
$$

for each $a \in A$, where the infimum is taken among all the representations $a=a_{0}+a_{1}$ with $a_{0} \in A_{0}$, $a_{1} \in A_{1}$.

Given $0<\alpha<1$, the (real) interpolation space $\left(A_{0}, A_{1}\right)_{\alpha, 2}$ is defined to be the set of functions $a \in A_{0}+A_{1}$ such that the norm

$$
\|a\|_{\left(A_{0}, A_{1}\right)_{\alpha, 2}}:=\left(\int_{0}^{\infty}\left|t^{-\alpha} K(t, a)\right|^{2} \frac{d t}{t}\right)^{1 / 2}
$$

is finite.

The definition above gives the standard interpolation between Sobolev spaces (see Chapter 2 in [12]). In particular we have that 
Theorem 3.3 (Theorem 2.4.2. in [12]). For any $\Omega$ bounded and $\mathcal{C}^{\infty}$ domain in $\mathbb{R}^{n}$. Fix real numbers $s_{0}, s_{1} \in \mathbb{R}$ and $0<\alpha<1$, satisfying

$$
s=(1-\alpha) s_{0}+\alpha s_{1}
$$

Then we have the following (real) interpolation equality

$$
\left(H^{s_{0}}(\Omega), H^{s_{1}}(\Omega)\right)_{\alpha, 2}=H^{s}(\Omega) .
$$

Proposition 3.4. Let

$$
\tilde{Y}:=\left\{f \in H^{r+2 \alpha}: \phi f \in H^{s+2 \alpha}\right\} .
$$

It holds that

$$
\|f\|_{\tilde{Y}} \leq\|f\|_{Y} \quad \text { for all } f \in Y
$$

and consequently, $Y \subset \tilde{Y}$.

Proof. Consider the Peetre's $K$-functional

$$
K(t, f)=\inf \left\{\left\|f_{0}\right\|_{X}+t\left\|f_{1}\right\|_{Z}\right\},
$$

where $f=f_{0}+f_{1}, f_{0} \in X, f_{1} \in Z$ and

$$
\begin{aligned}
& \left\|f_{0}\right\|_{X}=\left\|f_{0}\right\|_{H^{r}}+\left\|\phi f_{0}\right\|_{H^{s}}, \\
& \left\|f_{1}\right\|_{Z}=\left\|f_{1}\right\|_{H^{r+2}}+\left\|\phi f_{1}\right\|_{H^{s+2}} .
\end{aligned}
$$

Since inf $\left\{x_{i}+y_{j}\right\} \geq \inf \left(x_{i}\right)+\inf \left(x_{j}\right)$, we obtain that

$$
K(t, f) \geq K_{1}(t, f)+K_{2}(t, f),
$$

where $K_{1}$ is the Peetre's $K$-functional of the interpolation

$$
\left(H^{r}, H^{r+2}\right)_{\alpha, 2}=H^{r+2 \alpha},
$$

and $K_{2}$ the one corresponding to the interpolation

$$
\left(H_{\phi}^{s}, H_{\phi}^{s+2}\right)_{\alpha, 2}
$$

where we have defined the space

$$
H_{\phi}^{p}:=\left\{f: \phi f \in H^{p}\right\}
$$

We claim that

$$
\left(H_{\phi}^{s}, H_{\phi}^{s+2}\right)_{\alpha, 2} \subseteq H_{\phi}^{s+2 \alpha}
$$

To show it, let $f \in\left(H_{\phi}^{s}, H_{\phi}^{s+2}\right)_{\alpha, 2}$. Its norm can be computed with the formula (3.3) where the $K$ functional is given by

$$
K_{2}(t, f)=\inf \left\{\left\|\phi a_{0}\right\|_{H^{s}}+t\left\|\phi a_{1}\right\|_{H^{s+2}}\right\}
$$

where the inf is taken among all the pairs such that $a_{0}+a_{1}=f$. On the other hand, when we compute the $H^{s}$ norm of $\phi f$ we need to consider the functional

$$
K_{3}(t, \phi f)=\inf \left\{\left\|\bar{a}_{0}\right\|_{H^{s}}+t\left\|\bar{a}_{1}\right\|_{H^{s+2}}\right\}
$$

among all the pairs $\bar{a}_{0}, \bar{a}_{1}$ such that $\bar{a}_{0}+\bar{a}_{1}=\phi f$. It is clear then that $K_{2} \geq K_{3}$ because the infimum for $K_{3}$ is computed on a bigger set that may contain more pairs. 


\subsection{Construction of $\hat{Y}$}

This is the most delicate step in the characterization of the space $Y$. Our reference space $X$ is locally defined (it depends on the cutoff $\phi$ ), however, the fractional Laplacian is a non-local operator. The compromise between the local and non-local setting requires a different cutoff $\hat{\phi}$ such that supp $\hat{\phi} \supset \operatorname{supp} \phi$, as it was done in Section 2.

Proposition 3.5. Let $0<\alpha<1$ and $Y=(X, Z)_{\alpha, 2}$. Define the new function space $\hat{Y}$ as

$$
\hat{Y}:=\left\{f \in H^{r+2 \alpha}: \hat{\phi} f \in H^{s+2 \alpha}\right\}
$$

with $\hat{\phi}$ a smooth cut-off function such that $\hat{\phi}=1$ on the supp $\phi$, and norm

$$
\|f\|_{\hat{Y}}=\|f\|_{H^{r+2 \alpha}}+\|\hat{\phi} f\|_{H^{s+2 \alpha}} .
$$

It holds

$$
\hat{Y} \subset Y
$$

Proof. We need to show that, given any $f \in \hat{Y}$, then $(-\Delta)^{\alpha} f \in X$. It is clear that, since $f \in H^{r+2 \alpha}$, then $(-\Delta)^{\alpha} f \in H^{r}$.

The main problem is to understand the localization through the cutoff $\phi$, which means: suppose $(-\Delta)^{\alpha}(\hat{\phi} f) \in H^{s}$, prove that $\phi(-\Delta)^{\alpha} f \in H^{s}$.

In the next paragraph, we show that the operator $(-\Delta)^{\alpha}$ when considered on $\mathbb{R}$ or on the torus $\mathbb{T}^{1}$ is essentially the same, just by change of coordinates. This is an important property because it allows to prove the proposition using pseudo-differential calculus on $\mathbb{R}$.

The classical reference for the fractional Laplacian $(-\Delta)^{s}$ on $\mathbb{R}$ is [8]. Given $s \in(0,1)$ we define the fractional Laplacian as a pseudo-differential operator by

$$
\widehat{(-\Delta)^{s}} f(\xi)=|\xi|^{2 s} \hat{f}(\xi)
$$

i.e, its principal symbol is $|\xi|^{2 s}$. In [1], the authors have developed an equivalent definition using an extension problem to the half plane $\mathbb{R}_{+}^{2}$. For a function $f: \mathbb{R} \rightarrow \mathbb{R}$, we construct the extension $u:$ $\mathbb{R} \times[0,+\infty) \rightarrow \mathbb{R}, u=u(x, y)$, as the solution of the equation

$$
\left\{\begin{aligned}
\operatorname{div}\left(y^{1-2 \alpha} \nabla u\right) & =0 \quad \text { for } x \in \mathbb{R}, y \in[0,+\infty) \\
u(x, 0) & =f(x)
\end{aligned}\right.
$$

Then the fractional Laplacian of $f$ can be recovered, up to multiplicative constants, as

$$
(-\Delta)^{\alpha} f=\lim _{y \rightarrow 0} y^{1-2 \alpha} \partial_{y} u
$$

i.e., we are looking at a non-local Dirichlet-to-Neumann operator. Moreover, the extension $u$ can be written as $u=K *_{x} f$ where the Poisson kernel is defined by

$$
K(x, y)=\frac{y^{2 \alpha}}{\left(|x|^{2}+|y|^{2}\right)^{\frac{1+2 \alpha}{2}}}
$$

which means

$$
(-\Delta)^{\alpha} f=\int_{\mathbb{R}} \lim _{y \rightarrow 0} \partial_{y} K(x-\xi, y) f(\xi) d \xi
$$

Conformal transformations from the half-plane to the ball allow to transform the kernel $K$ from $\mathbb{R}$ to $\mathbb{T}^{1}$, and thus, the operator (3.7) as well. After the change of coordinates from the half plane to the ball, 
the operator (3.1) and (3.7) are equivalent.

Now we go back to the claim $\hat{Y} \subset Y$ for $x \in \mathbb{R}$, when the spaces are defined in $\mathbb{R}$. More precisely, we will show that given $(-\Delta)^{\alpha}(\hat{\phi} f) \in H^{s}(\mathbb{R})$, this implies $\phi(-\Delta)^{\alpha}(f) \in H^{s}(\mathbb{R})$. It holds

$$
\phi(-\Delta)^{\alpha}(f)=\phi(-\Delta)^{\alpha}(\hat{\phi} f)+G(f),
$$

where

$$
G f:=\phi\left[(-\Delta)^{\alpha}(f)-(-\Delta)^{\alpha}(\hat{\phi} f)\right]
$$

Take the $H^{s}$ norm, then

$$
\left\|\phi(-\Delta)^{\alpha}(f)\right\|_{H^{s}} \leq\left\|(-\Delta)^{\alpha}(\hat{\phi} f)\right\|_{H^{s}}+\|G(f)\|_{H^{s}}
$$

We will show that $G$ is a smoothing operator, i.e.,

$$
\|G(f)\|_{H^{s}} \leq C\|f\|_{H^{r+2 \alpha}}
$$

for $s>r+2 \alpha$, and this last norm is bounded by hypothesis.

We use some pseudo-differential operator tools to give information about $G$. Some standard references are $[10],[11],[6],[7]$. The advantage of working with pseudo-differential calculus in $\mathbb{R}$ is that the principal symbol of the fractional Laplacian is just $\sigma\left((-\Delta)^{\alpha}\right)=|\xi|^{2 \alpha}$. On the other hand, the principal symbol of the multiplication operator $M(f):=\hat{\phi} f$ is $\sigma(M)=\hat{\phi}(x)$.

Next, we compute the principal symbol of the composition operator $(-\Delta)^{\alpha}(\hat{\phi} \cdot)$. Its asymptotic expansion is given by

$$
\sigma\left((-\Delta)^{\alpha}(\hat{\phi} \cdot)\right)=\sum_{\theta \geq 0} \frac{1}{\theta !}\left(i D_{\xi}\right)^{\theta}|\xi|^{2 \alpha} D_{x}^{\theta} \hat{\phi} .
$$

The previous formula can be found, for instance, in [10], Chapter II, Section 4. In addition, the principal symbol for $\phi(-\Delta)^{\alpha}(\cdot)$ is just

$$
\sigma\left(\phi(-\Delta)^{\alpha}(\cdot)\right)=\phi(x)|\xi|^{2 \alpha}
$$

Then the principal symbol of the operator $G$ reduces to

$$
\sigma(G)= \begin{cases}0 & \text { on supp } \phi, \text { since } \hat{\phi}=1, \\ 0 & \text { otherwise. }\end{cases}
$$

This implies that the operator $G$ is zero modulus an operator with true order $-\infty$. We recall that the order of an operator $A$ is any number $q$ such that

$$
\|A u\|_{H^{p}} \leq\|u\|_{H^{p+q}}
$$

for each real $p$. The infimum of all such orders $q$ is called the true order of $G$.

Since the operator $G$ has true order $-\infty$, estimate (3.8) holds. In particular we have shown that

$$
\phi(-\Delta)^{\alpha}(f) \in H^{s}
$$

as we desired.

Remark: Consider now the function spaces $X$ and $Z$ defined as in Proposition 2.1

$$
X=H^{r} \cap H_{\phi}^{s}, \quad Z=H^{r+2} \cap H_{\hat{\phi}}^{s+2} .
$$

The interpolation space $Y:=(X, Z)_{\alpha, 2}$ is a subset of

$$
\tilde{Y}=\left\{f \in H^{r+2 \alpha}: \phi f \in H^{s+2 \alpha}\right\},
$$


and contains the space

$$
\bar{Y}=\left\{f \in H^{r+2 \alpha}: \bar{\phi} f \in H^{s+2 \alpha}\right\},
$$

where $\bar{\phi}$ is a cut-off function such that $\bar{\phi}=1$ on supp $\hat{\phi}$.

Aknowledgement M.d.M. Gonzalez is supported by Spain Government project MTM2008-06349-C03-01. M.P. Gualdani is supported by NSF-DMS 0807636. Support from IPAM, Institute of Pure and Applied Mathematics, UCLA is gratefully acknowledged.

\section{References}

[1] L. Caffarelli and L. Silvestre. An extension problem related to the fractional Laplacian. Comm. Partial Differential Equations, 32(7-9):1245-1260, 2007.

[2] L. Chayes, M. d. M. González, M. P. Gualdani, and I. Kim. A free boundary problem in price formation. Preprint, 2009 (arXiv:0902.3851).

[3] K.-J. Engel and R. Nagel. A short course on operator semigroups. Universitext. Springer, New York, 2006.

[4] M. d. M. González and M. P. Gualdani. Asymptotics for a free-boundary model in price formation. arXiv:0811.1636.

[5] D. Henry. Geometric theory of semilinear parabolic equations, volume 840 of Lecture Notes in Mathematics. Springer-Verlag, Berlin, 1981.

[6] L. Hörmander. Pseudo-differential operators. Comm. Pure Appl. Math., 18:501-517, 1965.

[7] J. J. Kohn and L. Nirenberg. An algebra of pseudo-differential operators. Comm. Pure Appl. Math., 18:269-305, 1965.

[8] N. S. Landkof. Foundations of modern potential theory. Springer-Verlag, New York, 1972. Translated from the Russian by A. P. Doohovskoy, Die Grundlehren der mathematischen Wissenschaften, Band 180.

[9] A. Pazy. Semigroups of linear operators and applications to partial differential equations, volume 44 of Applied Mathematical Sciences. Springer-Verlag, New York, 1983.

[10] M. Taylor. Pseudo differential operators. Lecture Notes in Mathematics, Vol. 416. Springer-Verlag, Berlin, 1974.

[11] M. E. Taylor. Pseudodifferential operators, volume 34 of Princeton Mathematical Series. Princeton University Press, Princeton, N.J., 1981.

[12] H. Triebel. Theory of function spaces, volume 78 of Monographs in Mathematics. Birkhäuser Verlag, Basel, 1983.

[13] H. Triebel. Theory of function spaces. II, volume 84 of Monographs in Mathematics. Birkhäuser Verlag, Basel, 1992.

[14] H. Triebel. Interpolation theory, function spaces, differential operators. Johann Ambrosius Barth, Heidelberg, second edition, 1995. 